\title{
Intervención con hombres penados por violencia contra la mujer en las relaciones de pareja: el Programa Contexto
}

\section{Intervention with intimate partner violence offenders: Contexto Program}

\author{
Fecha de recepción: 29/03/2016 \\ Fecha de aceptación: 12/06/2016
}

Raquel Conchell Diranzo

Profesora asociada del departamento de Psicología Social. Universidad de Valencia. Doctora en Psicología.

Marisol Lila Murillo

Profesora Titular del Departamento de Psicología Social de la Universidad de Valencia. Doctora en Psicología.

Alba Catalá-Miñana

Profesora post doctoral, Departamento de Psicología Social de la

Universidad Autónoma de Barcelona

\section{resumen/ahstract:}

Este trabajo describe la experiencia en la intervención con 643 hombres penados por violencia contra la mujer desde un modelo ecológico (Programa Contexto). Se analiza el perfil sociodemográfico de la muestra y la tasa de abandonos; se observa la evolución en variables relacionadas con la atribución de responsabilidad, la actitud hacia la violencia y las mujeres, y el ajuste psicosocial de los usuarios que han completado la intervención; se estudia cuál es la evolución en el riesgo de reincidencia de los usuarios que han completado la fase de seguimiento y se realiza una descripción sobre la tasa de reincidencia real según bases de datos oficiales. Los principales resultados indican que existe una baja tasa de abandonos y que tras la intervención se produce una reducción significativa en atribución de responsabilidad al sistema legal y la víctima, en sexismo hostil y benevolente y en sintomatología depresiva, así como un aumento de la gravedad percibida de la violencia y de la participación en la comunidad. Por último, se evidencia una disminución del riesgo de reincidencia y una baja tasa de reincidencia real. Los resultados muestran que el tratamiento ofrece cambios positivos.

This paper describes the experience in intervention with 643 men punished for violence against women, from an ecological model (Contexto Program). The demographic profile and dropout is analyzed; the change in variables related to attribution of responsibility, attitudes toward violence and women, and psychosocial adjustment of participants who have completed the intervention is observed; the change in recidivism risk of participants who have completed the follow-up phase is studied; and a description of the real rate of recidivism is made according to official databases. The main results indicate low dropout and significant reduction in several variables: attribution of responsibility to the legal system and the victim, hostile and benevolent sexism, and depressive symptoms; as well as increased the perceived severity of violence and community involvement. Finally, there is a decreased of recidivism risk and a low of real recidivism rate. The results show positive changes provided by the treatment.

\section{palabras clave/keywords:}

Atribución de responsabilidad, sexismo, reincidencia, programas de intervención con maltratadores, violencia contra la mujer.

Attribution of responsibility, sexism, recidivism, batterers intervention programs, intimate partner violence. 


\section{dossier}

\section{Introducción}

La violencia contra la mujer en las relaciones de pareja es uno de los tipos de agresiones interpersonales que más atención suscita. Este interés se centra principalmente en su etiología, en la multiplicidad de factores antecedentes, así como en la elevada prevalencia y morbilidad de sus efectos (Andrés-Pueyo y Echeburúa, 2010). Según la Organización Mundial de la Salud (OMS), ha llegado a convertirse en un problema de proporciones epidémicas (OMS, 2013). Muchos Estados, tras ser conscientes de la gravedad, han intentando buscar estrategias y herramientas para la solución de este grave problema social (Gondolf, 2012). En España, durante el año 2015 se realizaron 96.021 denuncias por violencia contra la mujer en las relaciones de pareja. En 2014, los órganos judiciales dictaron un total de 46.313 sentencias penales por violencia de género y el $61.2 \%$ supusieron una sentencia condenatoria. En 2015, el 15.5\% de las mujeres residentes en España de 16 años en adelante afirmaron haber sufrido violencia física, sexual o haber sentido miedo de alguna pareja o expareja en algún momento de su vida. Desde 1999, año en el que comienzan a recogerse de forma sistemática las cifras de mujeres muertas a manos de sus parejas o expareja, hasta el 2015, han muerto 1.043 mujeres (Ministerio de Sanidad, Servicios Sociales e Igualdad, 2015).

Una de las estrategias para la erradicación de la violencia contra la pareja es la intervención con hombres que ejercen la violencia de género. En España, comenzó esta iniciativa en 1995 con el programa Hombres que Ejercen Violencia en la Pareja (HEVPA), puesto en marcha en el País Vasco (Boira, 2010). Más tarde, tras la aprobación y entrada en vigor de la Ley Orgánica 1/2004, de Medidas de Protección Integral contra la Violencia de Género, se promovieron nuevas medidas de protección para las mujeres que padecen el maltrato de su pareja. Dicha ley, en su artículo 35, contempla la suspensión de la pena condicionada a la participación en programas de intervención con maltratadores. En España, a partir de este momento, ha habido un incremento de programas implementados para hombres penados por violencia de género (Arias, Arce y Vilariño, 2013; Lila, 2013). En la actualidad, existen diferentes tipos de programas de intervención. Por un lado, programas para agresores de violencia de género ingresados en prisión, por otro lado, programas de intervención en medio abierto para hombres penados por violencia contra la mujer con sustitución de su condena y, por último, y en menor medida, programas de intervención con hombres que no tienen ninguna condena y que voluntariamente asisten para ser ayudados (Boira, Carbajosa y Lila, 2014).

Los profesionales que trabajan en los programas de intervención con hombres penados por violencia contra la mujer indican que uno de los principales retos en este ámbito es mejorar la eficacia de estos programas y conseguir cambios en las actitudes hacia la violencia en los participantes (Boira, López, Tomás-Aragonés y Gaspar, 2013; Conchell, Lila y Gracia, 2013; Lila, 2013). Con el fin de mejorar la eficacia de este tipo de programas, han sido consideradas diversas variables relacionas con la violencia de género, entre las que se encuentran las actitudes hacia la violencia de género, los estilos de asunción de responsabilidad y el ajuste psicosocial (Conchell, Lila y Catalá-Miñana, 2012). 


\section{dossier}

\section{Indicadores de cambio en la intervención con hombres penados por violencia de género}

En la mayoría de los programas de intervención con hombres penados por violencia de género uno de los objetivos clave es que los participantes no atribuyan sus actos a factores externos, como el sistema judicial o la víctima, sino que asuman su responsabilidad y eliminen las justificaciones y las racionalizaciones que utilizan para el mantenimiento de su conducta violenta (Lila, Oliver, Catalá-Miñana, Galiana y Gracia, 2014).

Por otra parte, los estudios realizados desde una perspectiva ecológica muestran que el nivel de violencia contra la pareja está fuertemente asociado a los roles de género aceptados en cada país (Heise, 2011). Estas actitudes, si son tolerantes o favorables, pueden influir en el mantenimiento y fomento de dicha violencia (Gracia y Lila, 2015). Otro de los objetivos de intervención son los síntomas de desajuste psicológico. Los síntomas depresivos y la falta de apoyo social son algunas de las variables detectadas en el desajuste psicológico de este tipo de población. Diversos estudios han mostrado una mayor tendencia a la depresión en los hombres penados por violencia contra la mujer, por lo que algunos autores apuntan la necesidad de tratar este tipo de sintomatología para reducir el riesgo de reincidencia (Boira et al., 2014; Conchell et al., 2012). Por otro lado, la falta de apoyo social se relaciona con el desajuste psicológico y el comportamiento violento, incrementando la aparición y mantenimiento de conductas agresivas (Lila, Oliver, Lorenzo y Catalá, 2013).

El objetivo último de intervenir en variables como la atribución de responsabilidad, las actitudes hacia la violencia, el sexismo o el ajuste psicosocial, es reducir o eliminar la reincidencia. Para ello, es muy importante realizar una valoración del riesgo de reincidencia, ya que con su utilización se pueden evitar nuevas agresiones e incluso la muerte de la mujer (Andrés-Pueyo, 2009).

En base a lo expuesto, el objetivo de este trabajo es describir las características y principales resultados de la implementación de un programa de intervención con hombres penados por violencia de género y con una suspensión de condena. Los objetivos específicos son:

1. Analizar la evolución observada en variables relacionadas con la atribución de responsabilidad, las actitudes hacia la violencia y las mujeres, y el ajuste psicosocial de los usuarios que han completado la intervención $(n=402)$.

2. Analizar la evolución observada en el riesgo de reincidencia de los usuarios que han completado la fase de seguimiento $(n=50)$.

3. Describir la tasa de reincidencia real según bases de datos oficiales $(n=590)$.

\section{Método}

\section{Participantes}

La muestra de este estudio está compuesta por hombres penados por violencia contra la mujer en las relaciones de pareja que han sido derivados desde Servicios Sociales Penitenciarios al Programa Contexto, como medida alternativa a la pena de prisión. Para la admisión en el programa de intervención, los participantes deben cumplir con algunos requisitos, siendo los criterios de inclusión: 1) ser un hombre adulto (mayor de 18 años); 2) tener una 


\section{dossier}

suspensión de condena por violencia contra la mujer y la obligatoriedad de realizar un programa de intervención con maltratadores; 3) no tener trastornos de personalidad o psicopatologías graves; y 4) no sufrir grave adicción al alcohol u otras sustancias.

Entre los años 2006 y 2015, fueron atendidos 643 usuarios derivados desde Instituciones Penitenciarias al Programa Contexto. Actualmente, 50 usuarios se encuentran en fase de intervención. De los 593 usuarios que ya no se encuentran en fase de intervención, 191 (32.21\%) no completaron el tratamiento por diversos motivos: $50(8.43 \%)$ usuarios no acudieron al recurso a pesar de haber sido derivados como medida penal impuesta, $38(6.41 \%)$ fueron descartados para el proceso de intervención por no cumplir con los criterios de admisión, a $6(1.01 \%)$ se les revocó la medida penal impuesta, 13 (2.19\%) usuarios ingresaron en prisión durante el proceso de intervención, 9 (1.52\%) usuarios causaron baja por problemas de salud y no poder acudir a las sesiones, 13 (2.19\%) usuarios fueron dados de baja por comportamientos disruptivos y $62(10.46 \%)$ causaron baja por faltas de asistencia. Finalmente, 402 completaron la intervención (67.79\%).

El rango de edad comprende de los 18 a los 84 años $(M=39.54, D T=11.38)$. Respecto al país de nacimiento, la tasa de participantes nacidos en España fue 65.2\%, siendo el resto de participantes inmigrantes (34.8\%). El 39.8\% de los participantes estaban separados o divorciados, el $34.2 \%$ solteros y un $25.2 \%$ estaban casados. En cuanto al nivel de estudios, casi la mitad de los participantes que componen la muestra tenía estudios elementales o primarios (46.1\%), el 35.7\% tenía estudios secundarios, el 9.8\% contaba con estudios universitarios y el $8.3 \%$ no tenía estudios. En cuanto al estatus laboral, entre los usuarios existe un tasa del desempleo del $41.3 \%$. Aproximadamente el $81 \%$ de la muestra tiene unos ingresos anuales menores a 18.000 euros. Según los hechos probados de los participantes, el $70.6 \%$ de los usuarios habían sido penados por utilizar violencia física, mientras que el resto $(29.4 \%)$ fueron penados por utilizar algún tipo de violencia psicológica.

\section{Instrumentos}

Escala de Gravedad Percibida de la violencia contra la mujer en las relaciones de pareja (Gracia, García y Lila, 2011). Escala compuesta por ocho escenarios hipotéticos de violencia contra la mujer en las relaciones de pareja, que el penado tiene que valorar en función de su gravedad. Los escenarios están diseñados de forma que difieren en la severidad de la violencia. Incluyen violencia psicológica (e.g., "Una mujer es despreciada y humillada continuamente por su pareja"), amenazas (e.g.,"Una pareja discute, el hombre insulta a la mujer y amenaza con pegarle"), diferentes grados de violencia física (e.g., "En una discusión, un hombre pega a la mujer y después le pide perdón"), así como ejemplos de reincidencia (e.g.,"Una mujer es golpeada frecuentemente por su pareja, causándole a veces pequeñas lesiones y hematomas, aunque no quiere denunciar los hechos"). Se contesta en una escala de 0 (no es grave en absoluto) a 10 (la situación es muy grave). A mayor puntuación, mayor gravedad percibida de la situación $(\alpha$ pre-tratamiento $=.86 ; \alpha$ post-tratamiento $=.90)$.

Inventario de Sexismo Ambivalente (ASI). Versión española adaptada por Expósito, Moya y Glick (1998). Cuestionario que consta de 22 ítems con respuesta en escala tipo Likert de 6 puntos. Evalúa el sexismo ambivalente, definido como una ideología compuesta por prejuicios hacia la mujer. La escala está compuesta por dos factores: Sexismo Hostil (e.g., “

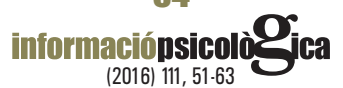




\section{dossier}

Las mujeres intentan ganar poder controlando a los hombres") $(\alpha$ pre-tratamiento $=.89 ; \alpha$ post-tratamiento $=.93$ ) y Sexismo Benevolente (e.g., "Aun cuando un hombre logre muchas cosas en su vida, nunca podrá sentirse verdaderamente completo a menos que tenga el amor de una mujer" $)(\alpha$ pre-tratamiento $=.84 ; \alpha$ post-tratamiento $=.81)$.

Escala de Atribución de Responsabilidad de la Violencia en las Relaciones de Pareja (IPVRAS) (Lila et al., 2014), compuesta por 12 ítems que evalúan dónde sitúan los penados por violencia contra la mujer la responsabilidad de la situación que los llevó a ser condenados. Se trata de una escala tipo Likert de 5 puntos, donde 1 significa "muy en desacuerdo" y 5 supone estar "muy de acuerdo". Esta escala está compuesta por tres factores: (1) Atribución de la responsabilidad al sistema legal, compuesto por 4 ítems que evalúan el grado de confianza de los participantes en el sistema legal (e.g., "Un sistema legal injusto es el responsable de que me encuentre en esta situación") ( $a$ Pre-tratamiento $=.64 ; a$ Posttratamiento $=.65) ;(2)$ Atribución de la responsabilidad al contexto personal del agresor, factor compuesto por 4 ítems que evalúan el grado en el que el participante atribuye la causa de los hechos a su situación personal (e.g., "La bebida o el uso de drogas es la causa de que me encuentre en esta situación") ( $a$ Pre-tratamiento $=.60 ; a$ Post-tratamiento $=.60) ;$ y (3) Atribución de la responsabilidad a la víctima, factor compuesto por 4 ítems que evalúan el grado en que el participante sitúa la culpa de su situación en las mentiras y/o en características de personalidad o conducta de la víctima (e.g., "El carácter agresivo, falta de control, nerviosismo o problemas psicológicos de mi pareja es la causa de que me encuentre en esta situación") ( $a$ Pre-tratamiento $=.62 ; a$ Post-tratamiento $=.70)$.

Escala de Síntomas Depresivos (CESD-7) (Herrero y Gracia, 2007). Se trata de una versión breve del CES-D (20 ítems) (Radloff, 1977) compuesta por los 7 ítems más eficaces para diferenciar las personas deprimidas de las no deprimidas. Pretende identificar niveles elevados de sintomatología depresiva en personas sin diagnóstico clínico (e.g., "Sentía como si no pudiera quitarme de encima la tristeza"). Se contesta en una escala de respuesta tipo Likert de 4 puntos, donde 1 es "rara vez o nunca (menos de un día)" y 4 significa "todo el tiempo o la mayoría del tiempo (5-7 días)". A mayor puntuación, mayor índice de riesgo de padecer depresión $(a$ Pre-tratamiento $=.86 ; a$ Post-tratamiento $=.87$ ).

Cuestionario de Apoyo Social Comunitario Percibido (ASC) (Gracia, Herrero y Musitu, 2002). Escala de 18 ítems que evalúa el apoyo que el participante percibe por parte de la comunidad, del entorno en el que vive. La escala está compuesta de 4 dimensiones en 3 escalas: la primera escala mide Integración Comunitaria (e.g, "Me siento identificado con mi comunidad") ( $a$ Pre-tratamiento $=.62 ; a$ Post-tratamiento $=.60)$ y Participación en la Comunidad (e.g., "Colaboro en las organizaciones y asociaciones de mi comunidad") ( $a$ Pre-tratamiento $=.77 ; a$ Post-tratamiento $=.76)$. La segunda escala mide Apoyo Social en los Sistemas Informales: asociaciones de vecinos, parroquia, agrupaciones políticas, asociaciones deportivas, etc. (e.g., "En estas organizaciones podría encontrar personas que me ayudaran a resolver mis problemas") ( $a$ Pre-tratamiento $=.85 ; a$ Post-tratamiento $=.87$ ); $\mathrm{y}$ la tercera mide Apoyo Social en los Sistemas Formales: centros educativos, centros de rehabilitación, etc. (e.g., "Si tuviera problemas -personales, familiares, etc.- podría encontrar personas en estas organizaciones que me ayudarían a resolverlos" $)(a$ Pre-tratamiento $=.73$; 


\section{dossier}

$a$ Post-tratamiento $=.70)$. Se trata de una escala tipo Likert de 5 puntos, donde 1 significa "totalmente en desacuerdo" y 5 supone estar "totalmente de acuerdo". A mayor puntuación obtenida en el cuestionario significa mayor apoyo comunitario percibido.

Escala de Valoración del Riesgo de Violencia contra la Pareja (SARA). Versión española adaptada por Andrés-Pueyo y López (2005). Es un protocolo clínico actuarial de 20 ítems cumplimentado por el profesional y con el que se valora el riesgo de reincidencia del agresor. Cada ítem se corresponde con un factor de riesgo y se cumplimenta a partir de la información obtenida a través de diversas fuentes (cuestionarios, entrevistas, etc.). El sumatorio de estos factores de riesgo indica que a mayor puntuación, mayor riesgo de reincidencia. Además de estos ítems, el instrumento incorpora dos indicadores: valoración general del riesgo de reincidencia contra la pareja y valoración del riesgo de reincidencia contra otras personas. En ambos casos el evaluador propondrá una valoración de riesgo (bajo $=0$, moderado $=1$ o alto $=2$ ). Para este estudio se utiliza el sumatorio de factores de riesgo y la valoración de riesgo contra la pareja.

\section{Reincidencia Real}

Se consultó la Base de datos VIOGEN (Sistema de seguimiento integral en los casos de violencia de género), elaborada desde el Ministerio del Interior, para comprobar la existencia de nuevos episodios de violencia por parte de los usuarios del programa $(0=$ no reincidente; $1=$ reincidente .

\section{El Programa}

El Programa Contexto es un programa de intervención para hombres penados por violencia contra la pareja, implementado en la Universidad de Valencia. Se basa en el modelo ecológico, recomendado por la Organización Mundial de la Salud (Heise, 2011). El objetivo principal del programa es aumentar los factores de protección y reducir los factores de riesgo para evitar la conducta violenta contra las mujeres en las relaciones de pareja, teniendo en cuenta cuatro niveles de análisis: individual, interpersonal, situacional y macrosocial (Catalá-Miñana, Walker, Bowen y Lila, 2014). El programa comienza con una fase de evaluación, con el objetivo de recopilar información, verificar el cumplimiento de los criterios de inclusión y aumentar la motivación para participar en el programa. Esta fase incluye la administración de una batería de pruebas estandarizadas y tres entrevistas motivacionales.

La fase de intervención consta de siete módulos distribuidos en 32 sesiones, que se realizan en 9 meses, con una sesión semanal de dos horas (10-12 participantes). Los participantes tienen que completar todo el programa para cumplir con la medida judicial impuesta. Se trata de una intervención grupal a largo plazo que cumple con los estándares recomendados en meta-análisis anteriores (Sánchez-Meca, Marín-Martínez y López-López, 2011). Los grupos son cerrados (no pueden entran nuevos participantes después del inicio del programa) y dos profesionales formados expresamente en violencia de género y un supervisor guían cada grupo. En cada módulo varía el número de sesiones. En el primer módulo (2 sesiones), la prioridad es la construcción de un clima de confianza en el trabajo de grupo, y establecer las normas de funcionamiento. En el segundo módulo (5 sesiones), se introducen los con- 


\section{dossier}

ceptos básicos. Este módulo también introduce por primera vez algunas de las actividades dirigidas a eliminar las distorsiones de los participantes (e.g., la negación, minimización, culpar a la víctima) y aumentar la responsabilidad de su propio comportamiento (esta es una tarea transversal que continúa durante toda la intervención). A partir del tercer módulo hasta el sexto, los objetivos de las sesiones son aumentar los factores de protección, proporcionar a los participantes los recursos y habilidades, así como la reducción de los factores de riesgo en cuatro niveles: individual (tercer módulo, 10 sesiones), interpersonal (cuarto módulo, 6 sesiones), situacional (quinto módulo, 2 sesiones), y sociocultural (sexto módulo, 5 sesiones). En el séptimo módulo (2 sesiones), las sesiones están destinadas a trabajar la prevención de la reincidencia y el fortalecimiento de las estrategias aprendidas. En la última sesión de intervención, se vuelve a administrar una batería de cuestionarios midiendo las mismas variables que en la fase pre-tratamiento. Después de completar la intervención, comienza la fase de seguimiento. Esta etapa tiene una duración de 18 meses a partir de la finalización del programa, con seis sesiones de seguimiento cada tres meses.

\section{Procedimiento}

Los participantes respondieron a una batería de cuestionarios dos veces durante la participación en la intervención (en las fases pre-tratamiento y post-tratamiento), con un intervalo de tiempo entre dichas fases de aproximadamente 11 meses. Fueron informados, en todo momento, de la naturaleza y el propósito de la investigación. El personal del programa administró los instrumentos y en los casos en los que había problemas respecto a la comprensión se les ayudó leyéndoles los ítems en voz alta individualmente. Adicionalmente, los profesionales encargados de la intervención evaluaron el riesgo de reincidencia en 8 momentos: en las fases pre-tratamiento y post-tratamiento y en los 6 seguimientos realizados tras finalizar la intervención (a los 3, 6, 9, 12, 15 y 18 meses). Debido a que la fase de seguimiento no está incluida en la medida penal y, por lo tanto, no es obligatoria, el tamaño de la muestra se ha visto reducido sustancialmente. Por último, debido a que no se cuenta con acceso permanente a las bases oficiales de reincidencia, no ha sido posible obtener dicha información de la muestra completa $(n=643)$, sino de 590 participantes.

\section{Resultados}

En relación al primer objetivo, para observar el efecto del tratamiento en los participantes, se compararon las puntuaciones obtenidas en fase pre y post-tratamiento de los 402 usuarios que finalizaron la intervención en atribución de la responsabilidad (atribución de la responsabilidad al sistema legal, a la víctima y al contexto personal), en actitud hacia la violencia y hacia las mujeres (gravedad percibida de la violencia contra la pareja, sexismo hostil y sexismo benevolente) y en ajuste psicosocial (sintomatología depresiva, integración en la comunidad, participación en la comunidad y percepción de apoyo social por parte de sistemas formales e informales) (ver Tabla1).

Las pruebas $t$ de muestras relacionadas evidenciaron que se produjo una reducción estadísticamente significativa en la atribución de la responsabilidad al sistema legal $(t=5.585 ; p<$ $.001 ; d=.29)$ y a la víctima $(t=6.409 ; p<.001 ; d=.33)$, en el sexismo hostil $(t=6.583 ; p$ 
Tabla 1.- Datos descriptivos de las variables de cambio $(n=402)$

\begin{tabular}{lcccc}
\hline & \multicolumn{2}{c}{ Pre-tratamiento } & \multicolumn{2}{c}{ Post-tratamiento } \\
\cline { 2 - 5 } & $\mathrm{M}$ & $\mathrm{DT}$ & $\mathrm{M}$ & $\mathrm{DT}$ \\
\hline IPVRAS $^{\text {a }}$ Sistema legal & 12.22 & 4.33 & 10.97 & 4.19 \\
IPVRAS $^{\text {a. }}$. Contexto personal & 7.37 & 3.56 & 7.32 & 3.42 \\
IPVRAS $^{\text {a }}$. Víctima & 13.07 & 4.46 & 11.59 & 4.58 \\
Gravedad percibida de la violencia & 70.35 & 12.04 & 72.17 & 12.01 \\
contra la pareja & & & & \\
Sexismo hostil & 2.53 & 1.17 & 2.11 & 1.19 \\
Sexismo benevolente & 2.93 & 1.11 & 2.42 & 1.11 \\
Sintomatología depresiva & 12.98 & 5.38 & 11.80 & 4.50 \\
ASC $^{b}$. Integración & 13.93 & 3.51 & 13.86 & 2.95 \\
ASC $^{b}$. Participación & 14.03 & 5.31 & 15.14 & 4.41 \\
ASC $^{b}$. Sistemas informales & 18.19 & 5.00 & 18.65 & 4.14 \\
ASC $^{b}$. Sistemas formales & 14.72 & 3.73 & 14.83 & 3.46 \\
\hline
\end{tabular}

a Escala de Atribución de Responsabilidad de la Violencia en las Relaciones de Pareja;

${ }^{\mathrm{b}}$ Apoyo Social Comunitario

$<.001 ; d=.36)$ y benevolente $(t=8.292 ; p<.001 ; d=.46)$ y en la sintomatología depresiva $(t=4.300 ; p<.001 ; d=.24)$. Además, se produjo un aumento en la gravedad percibida de la violencia contra la pareja $(t=-2.672 ; p<.01 ; d=.15)$ y en la participación en la comunidad $(t=-4.224 ; p<.001 ; d=.23)$. Sin embargo, no se produjeron diferencias estadísticamente significativas en atribución de la responsabilidad al contexto personal $(t=.289 ; p=.773)$, en integración en la comunidad $(t=.435 ; p=.664)$ ni en la percepción de apoyo social por parte de sistemas formales $(t=-.513 ; p=.608)$ e informales $(t=-1.580 ; p=.115)$.

En relación al segundo objetivo, una prueba $t$ de muestras relacionadas con el sumatorio de factores de riesgo de reincidencia como variable dependiente indica que, tras realizar la intervención, la acumulación de estos factores ha sido reducida de 8.10 de media $(D T=$ $5.15)$ a $5.92(D T=4.22)$ tras completar la intervención $(t=12.488 ; p<.001 ; d=.46)$. Por otro lado, en la valoración de riesgo realizada por los profesionales antes y después de la intervención, se puede observar cómo el porcentaje de usuarios calificados de riesgo alto se ve disminuido del $10.5 \%$ al $3.8 \%$, los calificados de riesgo moderado disminuyen del $36.4 \%$ al $26.9 \%$ y, por último, los calificados de riesgo bajo aumentan del $53.1 \%$ al $68.2 \%$ de la muestra.

Respecto a la evolución de la acumulación de factores de riesgo a lo largo de 18 meses de seguimiento, por el momento se ha obtenido una submuestra de 50 participantes, cuya evolución se puede observar en la Figura 1. Los datos descriptivos nos muestran cómo la reducción en riesgo de reincidencia, en general, se mantiene a lo largo del tiempo (ver Tabla 2). 


\section{dossier}

Tabla 2.- Datos descriptivos de la evolución del riesgo de reincidencia a lo largo de la fase de intervención y seguimiento $(n=50)$

\begin{tabular}{lcc}
\cline { 2 - 3 } & $\mathrm{M}$ & $\mathrm{DT}$ \\
\hline Pre-tratamiento & 8.9 & 5.23 \\
Post-tratamiento & 6.39 & 3.76 \\
Seguimiento 3 meses & 5.94 & 3.84 \\
Seguimiento 6 meses & 5.58 & 3.27 \\
Seguimiento 9 meses & 4.91 & 3.62 \\
Seguimiento 12 meses & 6.62 & 3.63 \\
Seguimiento 15 meses & 5.16 & 3.15 \\
Seguimiento 18 meses & 5.38 & 3.42 \\
\hline
\end{tabular}

Figura 1.- Evolución del riesgo de reincidencia en la fase de intervención y seguimiento $(n=50)$

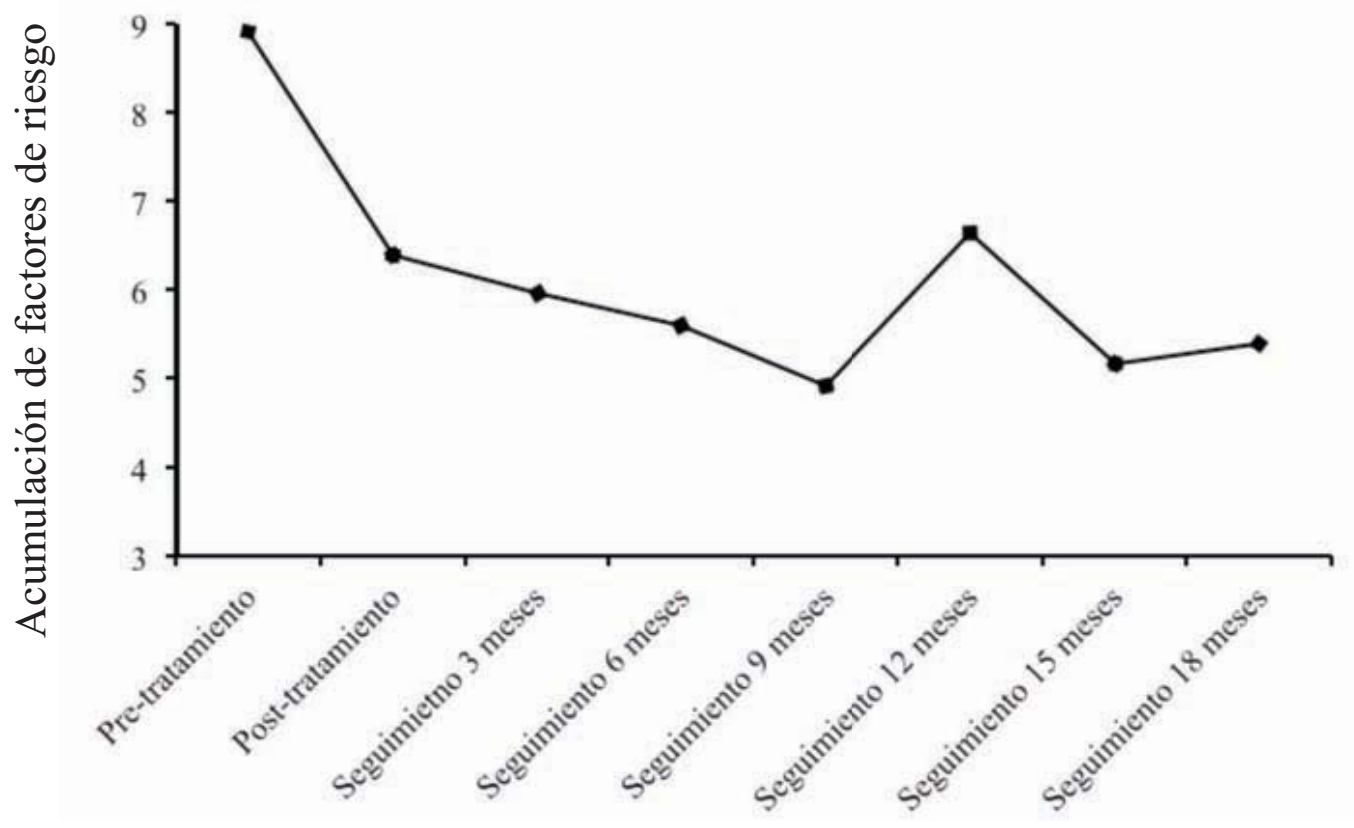


Por último, en cuanto a la reincidencia real comprobada, según las actualizaciones obtenidas hasta el 18 de febrero de 2015, se ha podido comprobar que 64 usuarios derivados al Programa Contexto de 590 usuarios contrastados en las bases de VIOGEN, han reincidido en delitos relacionados con la violencia de género (10.85\%). Específicamente, de los 402 usuarios que han finalizado la intervención, 38 han reincidido $(9.45 \%)$, y de los 50 usuarios que han completado los 18 meses de seguimiento, han reincidido 2 (4\%).

\section{Discusión y Conclusiones}

El objetivo general de este trabajo era describir las características y principales resultados de la implementación de un programa de intervención con hombres penados por violencia de género y con una suspensión de condena. Específicamente, los objetivos eran: 1) analizar la evolución observada en variables relacionadas con la atribución de responsabilidad, las actitudes hacia la violencia y las mujeres, y el ajuste psicosocial de los usuarios que han completado la intervención; 2) analizar la evolución observada en el riesgo de reincidencia de los usuarios que han completado la fase de seguimiento; y por último, 3) describir la tasa de reincidencia real según bases de datos oficiales.

En cuanto a las características sociodemográficas de los participantes que asisten al programa de intervención para hombres penados por violencia contra la mujer, se observa que, aunque no se puede hablar de un perfil específico en esta muestra, los datos apuntan en la dirección de otros estudios, que evidencian la existencia de características relacionadas con estas variables y que pueden suponer factores de riesgo para ejercer la violencia contra la mujer, por lo que han de ser tenidas en cuenta (Conchell et al., 2013). En este caso, se ha detectado un alto porcentaje de participantes que cuentan con estudios elementales o primarios (46.1\%), que el $43.3 \%$ están desempleados o que el 35\% son inmigrantes.

Además, se observa que las bajas producidas durante el transcurso de la intervención se debe a razones muy diversas y que la tasa de abandono se reduce a un $10.46 \%$. Incluso con los obstáculos surgidos por diferentes motivos (problemas de salud, problemas de de conducta, ingreso es prisión, etc.) el $67.79 \%$ consiguió finalizar la intervención.

Respecto al primer objetivo, podemos corroborar que se produce una disminución significativa en la atribución de la responsabilidad al sistema legal y a la víctima, una reducción del sexismo hostil y benevolente, una reducción de la sintomatología depresiva, y un aumento en la gravedad percibida de la violencia contra la pareja y en la participación en la comunidad. En relación a la atribución de la responsabilidad, los datos indican que tras la intervención los participantes externalizan menos la responsabilidad del hecho por el que han sido condenados, y esta modificación de su actitud es necesaria para que se produzca el cambio. La asunción de responsabilidad es clave como motivador para el cambio y se trata de un factor protector muy importante para reducir la probabilidad de reincidencia a corto y largo plazo (Conchell et al., 2012; Lila et al., 2014). Además se observan, como se ha indicado, cambios en actitudes relacionadas con la percepción de la violencia de género y en las actitudes sexistas. Estos resultados indican que tras la intervención son más conscientes de la diversidad de conductas que supone ejercer la violencia y las consecuencias de llevarlas a cabo (Conchell et al., 2012; Gracia y Lila, 2008). El hecho de ser capaz de reconocer qué 


\section{dossiert}

es violencia de género y la propia reducción de actitudes sexistas son importantes factores que disminuyen las probabilidades de ejercer la violencia (Heise, 2011). En cuanto a las variables de ajuste psicológico, numerosos estudios han mostrado una elevada prevalencia de la sintomatología depresiva en los participantes de los programas de intervención con hombres penados por violencia contra la mujer (Boira et al., 2014). En este sentido, algunos autores apuntan que fomentar el ajuste psicológico debería ser un objetivo en los programas de intervención con maltratadores (Lila et al., 2013). Parece que el Programa Contexto ayuda a favorecer dicho ajuste. En cuanto a los cambios en el apoyo social comunitario percibido se observa un aumento significativo de los niveles de participación. Actualmente, los investigadores de este ámbito indican que el entorno social es una variable clave en los programas de intervención con hombres penados por violencia contra la mujer (Lila et al., 2013). Este resultado es relevante, ya que los agresores que finalizan la intervención participan más en su comunidad, mostrando una intención de integrarse y conectarse con su entorno social. Este hecho supone fomentar el apoyo social en el proceso de cambio como uno de los factores protectores contra la violencia (Boira et al., 2014; Conchell et al., 2012).

Por otro lado, la atribución de la responsabilidad al contexto personal de los hechos ocurridos, la integración en la comunidad y la percepción de apoyo social por parte de sistemas formales e informales no han cambiado a lo largo de la intervención de forma estadísticamente significativa. Aunque los datos indican que la variación apunta en la dirección esperada, las mejoras en el diseño de intervención de este programa deberían ir encaminadas a potenciar más los cambios en este tipo de variables.

En relación al segundo objetivo, es importante destacar que la disminución del riesgo de reincidencia es uno de los principales elementos que se utiliza como indicador de éxito en los programas de intervención con hombres agresores. En este sentido, parece que los resultados obtenidos muestran que el paso por el programa de intervención supone una reducción del riesgo de reincidencia. Además, se observa que esta reducción tiende a mantenerse en el tiempo, cuestión clave de cara a la protección de posibles futuras parejas de los usuarios. Este tipo de valoración cada vez es más utilizada, ya que nos aporta datos de estimación fiables sobre la violencia a medio y largo plazo y además nos ayuda a tomar decisiones sobre el riesgo de reincidencia de los participantes del programa e individualizar estrategias en cada caso (Andrés-Pueyo, 2009).

Sin embargo, de forma generalizada se considera que el indicador más fiable es la comprobación de la reincidencia real (Pérez y Martínez, 2010). Es primordial poder analizar las cifras oficiales de reincidencia de los participantes que asisten a los programas de intervención con hombres penados por violencia contra la mujer. En este caso, los datos se han obtenido a través de la base VIOGEN elaborada desde el Ministerio del Interior. Esta base informa de la existencia de tasas de reincidencia en torno al 9.45\%, de manera similar a otros estudios nacionales e internacionales (Coulter y VandeWeerd, 2009; Pérez y Martínez, 2010). Esto supone una reducción de la reincidencia significativa, si se compara con el 20\%-30\% de reincidencia de hombres que agreden a la pareja de manera generalizada, según estudios previos (Pérez y Martínez, 2010). Aunque se trata de un bajo nivel de reincidencia, estos datos han de ser interpretados con cautela, ya que el hecho de que no se haya 


\section{dossier}

detectado de manera oficial, no quiere decir que no se estén dando casos de violencia. Además, observamos que la tasa de reincidencia se reduce más de la mitad cuando los usuarios completan la fase de seguimiento de 18 meses. Hay que tener en cuenta que el tamaño de la muestra en la fase de seguimiento es muy reducida y que es de carácter voluntario. El hecho de contar solamente con la información de los usuarios que están motivados a seguir acudiendo en la fase voluntaria, puede suponer un sesgo en los resultados. Por esta razón, uno de los objetivos de intervención debería ser aumentar la motivación y la adherencia al seguimiento, de manera que lleguen a completar esta fase un mayor número de participantes. Finalmente, a pesar de las dificultades encontradas para constatar la reincidencia real, la valoración de los resultados en conjunto (la escasa tasa de reincidencia real y la reducción tanto de los factores de riesgo como del riesgo de reincidencia), permite aportar evidencia de que el programa es eficaz y se dan cambios positivos y significativos en los participantes que completan la intervención.

Aunque los datos son alentadores, es importante continuar profundizando en las variables que son clave en la violencia contra la mujer en las relaciones de pareja. Concretamente, este estudio ofrece evidencia de que la intervención con hombres penados por este tipo de violencia puede fomentar cambios positivos relacionados con la reducción o eliminación de dicha violencia. Sin embargo, este trabajo no está exento de limitaciones. Por una parte, aunque la muestra es considerable en un principio, la pérdida de la información de los participantes que no completan la intervención supone que estos datos no sean generalizables a todos los usuarios del programa, y no es posible saber cuál es la evolución de los participantes que no consiguen adherirse al tratamiento. En la misma línea, el hecho de que la fase de seguimiento sea de carácter voluntario, hace que la pérdida de muestra sea considerable. Sin embargo, a pesar de que esta cuestión puede suponer un sesgo en los datos, los resultados siguen indicando que el tratamiento aporta aspectos positivos de cara a la reducción de la reincidencia. Por último, sería conveniente en futuros estudios comparar los resultados con una muestra de hombres agresores que no hayan recibido tratamiento.

\section{Referencias}

Andrés-Pueyo, A. y Echeburúa, E. (2010). Valoración del riesgo de violencia: instrumentos disponibles e indicaciones de aplicación. Psicothema, 22, 403-409.

Andrés-Pueyo, A. (2009). La predicción de la violencia contra la pareja. En E. Echeburúa, J. Fernández-Montalvo y P. de Corral (Eds.), Predicción del riesgo de homicidio y de violencia grave en la relación de pareja (pp. 21-56). Valencia: Centro Reina Sofía.

Andrés-Pueyo, A. y López, S. (2005). Manual para la valoración del riesgo de violencia contra la pareja. Barcelona: Publicacions i Edicions de la Universitat de Barcelona.

Arias, E., Arce, R. y Vilariño, M. (2013). Batterer intervention programmes: A meta-analytic review of effectiveness. Psychosocial Intervention, 22, 153-160.

Boira, S., Carbajosa, P. y Lila, M. (2014). Principales retos en el tratamiento grupal de los hombres condenados por un delito de violencia de género. Clínica Contemporánea, 5, 3-15.

Boira, S., López, Y., Tomas-Aragonés, L. y Gaspar, A. R. (2013). Efficacy of different treatment modalities in men convicted of intimate partner violence. Anales de Psicología, 29, 19-28. 
Boira, S. (2010). Hombres maltratadores. Historias de violencia masculina. Zaragoza: Prensas Universitarias de Zaragoza.

Catalá-Miñana, A., Walker, K., Bowen, E. y Lila, M. (2014). Cultural differences in personality and aggressive behavior in intimate partner violence offenders: A comparison of English and Spanish offenders. Journal of Interpersonal Violence, 29, 2652-2669.

Conchell, R., Lila, M. y Gracia, E. (2013). Intervención en medio abierto. Hombres penados por violencia de pareja. Un análisis pre-post de los indicadores de eficacia del Programa Contexto. Saarbrücken, Alemania: Publicia.

Conchell, R., Lila, M. y Catalá-Miñana, A. (2012). Cambios psicosociales en un programa de intervención con hombres penados por violencia contra la mujer. Revista de Psicología, 21, 159-186.

Coulter, M. y VandeWeerd, C. (2009). Reducing domestic violence and other criminal recidivism: Effectiveness of a multilevel batterers intervention program. Violence and Victims, 24, 139-152.

Expósito, F., Moya, M. y Glick, P. (1998). Sexismo ambivalente: medición y correlatos. Revista de Psicología Social, 55, 893-905.

Gracia, E. y Lila, M. (2015). Attitudes towards violence against women. Luxembourg: Publication Office of the European Union.

Gracia, E., García, F. y Lila, M. (2011). Police attitudes towards policing partner violence against women: Do they correspond to different psychosocial profiles?. Journal of Interpersonal Violence, 12, 648-656.

Gracia, E. y Lila, M. (2008). Los profesionales de la salud y la prevención de la violencia doméstica contra la mujer. Revista Médica de Chile, 136, 394-400.

Gracia, E., Herrero, J. y Musitu, G. (2002). Evaluación de recursos y estresores psicosociales en la comunidad. Madrid: Editorial Síntesis.

Gondolf, E. (2012). The future of batterer programs: Reassessing evidence-based practice. Boston: Northeastern University Press.

Heise, L. (2011). What works to prevent partner violence? An evidence overview. Working paper. London: Department for International Development.

Herrero, J. y Gracia, E. (2007). Una medida breve de la sintomatología depresiva (CES-D 7). Salud Mental, 30, 40-46.

Ley Orgánica 1/2004, de 28 de diciembre, de Medidas de Protección Integral contra la Violencia de Género. B.O.E. núm. 313, de 29 de diciembre de 2004.

Lila, M. (2013). La intervención con hombres condenados por violencia de pareja contra la mujer en España: Investigación y avances en intervención. Psychosocial Intervention, 22, 81-85.

Lila, M., Oliver, A., Catalá-Miñana, A., Galiana, L. y Gracia, E. (2014). The Intimate Partner Violence Responsibility Attribution Scale (IPVRAS). European Journal of Psychology Applied to Legal Context, 6, 29-36.

Lila, M., Oliver, A., Lorenzo, M. V. y Catalá, A. (2013). Valoración del riesgo de reincidencia en violencia contra la mujer en las relaciones de pareja: Importancia del apoyo social. Revista de Psicología Social, 28, 225- 236.

Ministerio de Sanidad, Servicios Sociales e Igualdad (2015). Macroencuesta sobre la violencia contra las mujeres. Madrid: Ministerio de Sanidad, Servicios Sociales e Igualdad.

Organización Mundial de la Salud (2013). Global and regional estimates of violence against women: Prevalence and health effects of intimate partner violence and non-partner sexual violence. Ginebra, Suiza: Organización Mundial de la Salud.

Pérez, M. y Martínez, M. (2010). La reincidencia de los condenados por delitos de violencia de género a programas formativos aplicados desde la ejecución penal en la comunidad. Barcelona: Centro de Estudios Jurídicos y Formación Especializada.

Radloff, L. S. (1977). The CES-D Scale: A self-report depression scale for research in the general population. Applied Psychological Measurement, 1, 385-401.

Sánchez-Meca, J., Marín-Martínez, F. y López-López, J. A. (2011). Meta-análisis e intervención psicosocial basada en la evidencia. Psychosocial Intervention, 20, 95-107. 